\title{
Urinary excretion of 1,5-anhydro-D-glucitol accompanying glucose excretion in diabetic patients
}

\author{
Y.Akanuma ${ }^{1}$, M. Morita ${ }^{2}$, N.Fukuzawa ${ }^{1}$, T. Yamanouchi $^{3}$ and H. Akanuma ${ }^{2}$ \\ ${ }^{1}$ Institute for Diabetes Care and Research, Asahi Life Foundation, \\ ${ }^{2}$ Department of Chemistry, College of Arts and Sciences, University of Tokyo, \\ ${ }^{3}$ The Second Department of Internal Medicine, University of Teikyo, Tokyo, Japan
}

\begin{abstract}
Summary. The urinary excretion of 1,5-anhydro-D-glucitol, a pyranoid polyol, in humans was studied. The plasma of nondiabetic human subjects contained high concentrations of this polyol $(>110 \mu \mathrm{mol} / \mathrm{l})$, and there was a tendency for the 24-h excretion of it to become more variable in direct proportion to its plasma concentration. In contrast, diabetic patients showed lower plasma concentrations of this polyol, and the variation in the 24-h excretion of 1,5-anhydro-D-glucitol was especially notable among the patients with an extremely low plasma concentration of the polyol. This diabetic group showed a statistically significant correlation $(p<0.01)$, between the urinary 1,5-anhydro-D-glucitol and urinary
\end{abstract}

glucose. This correlation was more markedly demonstrated during a 100 -g oral glucose tolerance test: parallel changes were observed in the concentrations of 1,5-anhydro-D-glucitol and glucose in the urine collected every hour after the glucose load. These observations led to the proposal that low plasma concentration of this polyol, which is observed in diabetes mellitus, may be the result of a frequent and/or prolonged high blood glucose concentration beyond the renal threshold for glucose excretion.

Key words: 1,5-anhydroglucitol, urinary excretion, reabsorption, glucosuria, hyperglycaemia.
A pyranoid polyol resembling glucose, 1,5-anhydro-Dglucitol (AG), is a humoral component in humans and other mammals [1-12]. The plasma AG concentration is specifically reduced in diabetes mellitus [10] and shows several dynamic properties which are advantageous as a clinical marker for diabetes mellitus. First, the extent of the plasma $A G$ reduction is apparently correlated with the severity of diabetes mellitus, and strict metabolic control can reverse this reduction [11]. Second, AG undergoes very slow turnover in vivo $[8$, 12], and its plasma concentration remains stable over a 24-h period, showing little acute response to fasting, food intake, and insulin administration [11]. Finally, AG also shows chemical and biochemical stability [12], and accordingly, we found no significant limitation on the storage and transport of plasma samples for $A G$ determination. On the other hand, the utility of the plasma AG concentration as a clinical marker is certainly limited by a lack of metabolic knowledge: the origin and clearance mechanism of AG in humans have not yet been established. Recently, we demonstrated the primary role of renal AG reabsorption in $A G$ retention in rats and mice [12]. That study also indicated a possibility that the diabetic plasma AG reduction is due to a decrease in renal AG reabsorption. In order to evaluate in humans the role of renal func- tion in $A G$ retention in the circulation, we measured the urinary AG and urinary glucose as well as the plasma AG and blood glucose.

\section{Subjects and methods}

\section{Subjects}

Study 1. The amounts of AG and glucose in the blood and in a 24-h urine sample were determined for 11 non-diabetic volunteers and 30 diabetic patients, which included two patients with Type 1 (insulin-dependent) diabetes mellitus while other patients had Type 2 (non-insulin-dependent) diabetes mellitus. Various characteristics of these diabetic patients are summarised in Table 1. The diabetic subjects were selected from hospitalised patients whose pooled 24-h urine was available and who showed large individual differences in the plasma $\mathrm{AG}$ concentration. Venous blood and plasma samples were obtained on the same day when urine was collected.

Study 2. The changes in the concentrations of plasma and urinary AG and blood and urinary glucose after a $100-\mathrm{g}$ oral glucose load were studied in five non-diabetic subjects, seven impaired glucose tolerance (IGT) patients and five diabetic patients. Blood samples were collected from these patients before and at 30,60, 90, 120 and $180 \mathrm{~min}$ after the glucose load, while urine specimens were collected before and at 60,120 , and $180 \mathrm{~min}$.

Diagnoses of diabetes and IGT were made according to the criteria established by the World Health Organization Expert Committee [13]. 
Table 1. Distributions of age, duration of diabetes, plasma and urinary AG, and plasma creatinine in control subject and diabetic patient groups

\begin{tabular}{llllll}
\hline Group & $\begin{array}{l}\text { Number of } \\
\text { subjects } \\
(\mathrm{F})\end{array}$ & $\begin{array}{l}\text { Age } \\
\text { (years) }\end{array}$ & $\begin{array}{l}\text { Duration of } \\
\text { diabetes } \\
\text { (years) }\end{array}$ & $\begin{array}{l}\text { Plasma AG } \\
(\mu \mathrm{mol} / 1)\end{array}$ & $\begin{array}{l}\text { Urinary AG } \\
(\mu \mathrm{mol} / \mathrm{day})\end{array}$ \\
\hline $\begin{array}{l}\text { Control } \\
\text { subjects }\end{array}$ & $11(0)$ & $\begin{array}{l}35.9 \pm 6.9 \\
(26-54)\end{array}$ & - & $\begin{array}{l}\text { Plasma } \\
\text { creatinine } \\
(\mu \mathrm{mol} / 1)\end{array}$ \\
$\begin{array}{l}\text { Diabetic } \\
\text { patients }\end{array}$ & $30(11)$ & $\begin{array}{l}54.6 \pm 10.4 \\
(25-72)\end{array}$ & $\begin{array}{l}12.1 \pm 10.5 \pm 39.1 \\
(116-252)\end{array}$ & $\begin{array}{l}28.2 \pm 13.8 \\
(12.7-58.5)\end{array}$ \\
\hline
\end{tabular}

Plus-minus values are mean \pm SD. Numbers in parentheses in the subject column indicate the number of females while those in other columns indicate ranges.

a 1,5-anhydro-D-glucitol

\section{Methods}

The glucose concentration in the blood and urine was determined using a FUJI DRI-CHEM 2000 analyzer (Fuji Photo Film Co., Tokyo, Japan) [14]. The plasma AG concentration was determined by gas-liquid chromatography, as described previously [11]. The urinary $\mathrm{AG}$ concentration was determined by a recently elaborated method using gas chromatography/mass spectrometry [12]. Briefly, $0.558 \mathrm{nmol}\left(100 \mathrm{ng}\right.$ ) of 1,5-anhydro-D-glucitol-1,2,3,4,6,6- ${ }^{2} \mathrm{H}_{2}$ (AG$d_{6}$ ) was first added to the urine sample $(100 \mu l)$; this served as the internal standard in the present mass spectrometric analysis. Then the urine was treated on a three-layer column containing, from the bottom, $\mathrm{H}$-form ( $1 \mathrm{ml}$ ) of a cation exchanger (AG 50W X8, Bio-Rad Laboratories, Richmond, Calif, USA) and $\mathrm{OH}$-form $(5 \mathrm{ml})$ and borate form $(0.5 \mathrm{ml})$ of an anion exchanger (AG 1X8, Bio-Rad Laboratories), using water as the eluent. The flow-through fraction $(5 \mathrm{ml})$ was dried and the resulting residue was acetylated for gas chromatography/mass spectroscopy analysis using a selected ion monitoring method in which $\mathrm{M} / \mathrm{Z}=212\left(\mathrm{M}^{+}-2 \mathrm{CH}_{3} \mathrm{COOH}\right)$ and 217 $\left(\mathrm{M}^{+}-\mathrm{CH}_{3} \mathrm{COOH}-\mathrm{CH}_{3} \mathrm{COO}^{2} \mathrm{H}\right)$ represented $\mathrm{AG}$ and $\mathrm{AG}-\mathrm{d}_{6}$ respectively. The relative amounts of $A G$ and $A G-d_{6}$ were calculated from the peak areas of the two specific fragments and the AG content of the original sample was deduced by referring to the AG- $d_{6}$ recovery.

\section{Statistical analysis}

The correlation coefficient between the plasma $A G$ and the urinary AG was determined by Spearman's coefficient $\left(r^{\prime}\right)$ of rank correlation method, and the relationship between the urinary glucose and the urinary AG was assessed by Pearson's simple correlation coefficient $(r)$. The results of the oral glucose load test were expressed as mean $\pm S D$. Within each group, the statistical significance of changes from the mean fasting value was evaluated at each timepoint by Student's paired $t$-test.

\section{Results}

\section{4-h urinary $A G$ of control subjects and diabetic patients}

The plasma AG concentration was generally low in the diabetic patients: it was less than $90 \mu \mathrm{mol} / 1$ in 28 of 30 diabetic patients, but more than $110 \mu \mathrm{mol} / 1$ in the control subjects (Fig. 1). It may be worth noting that the two diabetic patients with a plasma concentration of more than $90 \mu \mathrm{mol} / 1$ were apparently diabetic on the basis of the results of the glucose tolerance test. In

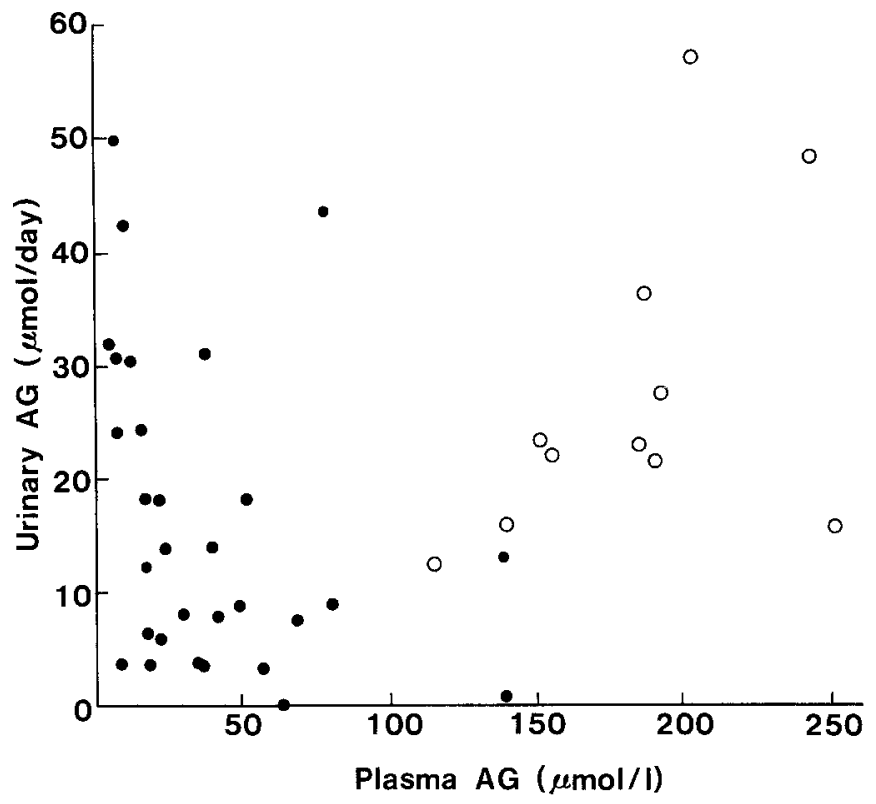

Fig. 1. Scattergram of 24-h urinary AG vs plasma AG concentration in patients with diabetes mellitus $(\Theta)$ and non-diabetic subjects $(O)$. The two AG values were inversely correlated in the diabetic group $\left(r^{\prime}=-0.408, p<0.05\right)$, while it was not indicated to be significant in the control group $\left(r^{\prime}=0.473, p>0.1\right)$ probably due to a small sample size

contrast to the marked difference between the two groups in the plasma AG concentration, a variable $A G$ amount was observed in the 24 -h urine of both normal and diabetic subjects. The blood AG concentration was surmised to affect the urinary AG concentration differently in the normal and diabetic groups (Fig. 1). On the one hand, the urinary AG amounts were more variable and larger on average for the control subjects who had a higher plasma AG concentration. On the other hand, this relationship between plasma and urinary AG was reversed in the diabetic group: the hospitalised diabetic patients with relatively high plasma AG concentrations showed the lowest urinary AG values, while some patients with extremely low plasma $A G$ values showed high urinary $A G$ values.

In the diabetic group, the 24-h urinary AG showed an appreciable correlation ( $r=0.721)$ with the 24 -h urinary glucose (Fig. 2). 


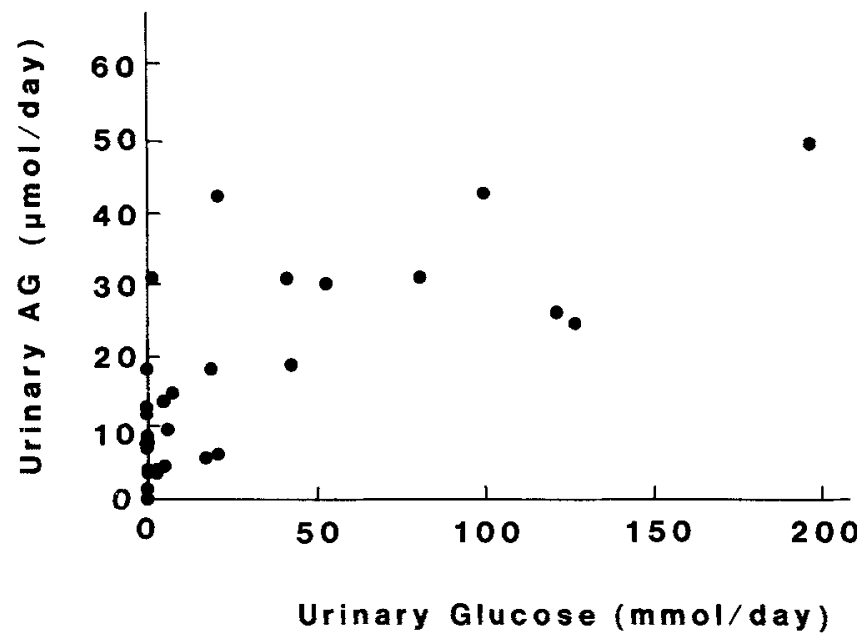

Fig. 2. Correlation between $A G$ and glucose in 24-h urine of diabetic patients. The data shown in this figure are from the data sets for the same diabetic patients shown in Figure 1. The correlation coefficient for the scattergram was calculated to be $0.721(p<0.01)$

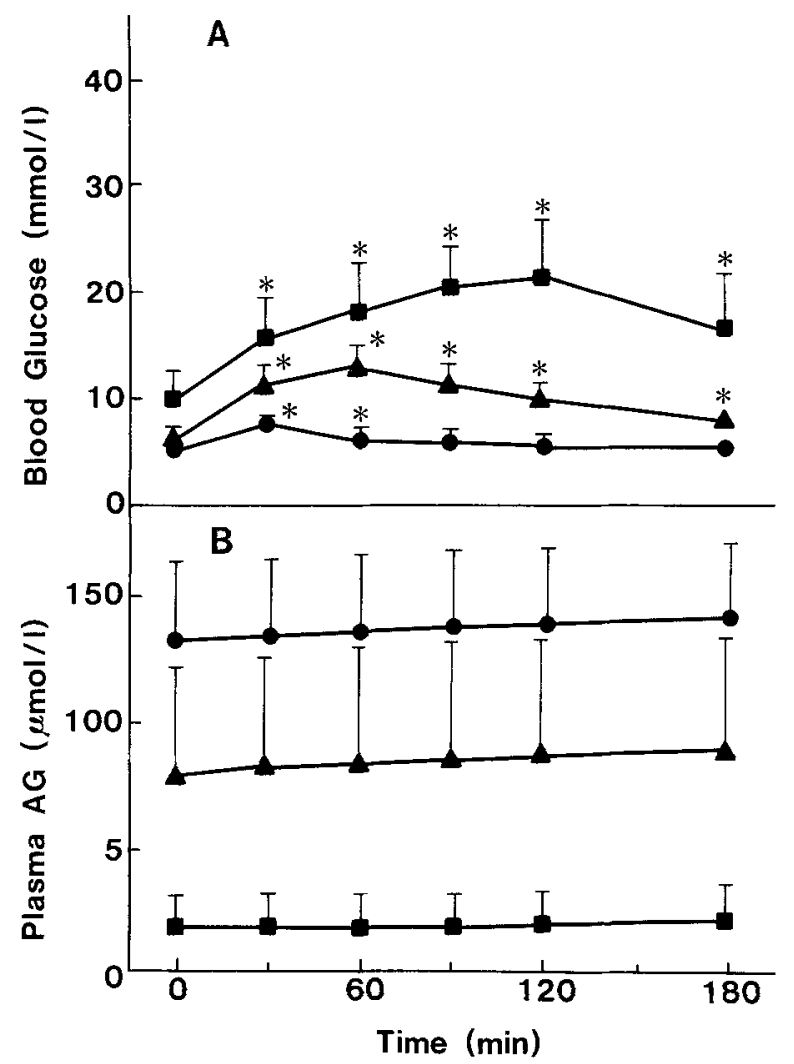

Fig. 3. Effects of $100 \mathrm{~g}$ glucose load on the concentrations of blood glucose (A) and plasma AG (B). Each plot represents the average values for the diabetic $(n=5,-\mathbf{\square}-)$, IGT $(n=7,-\boldsymbol{\Delta}-)$ and non-diabetic $(n=5,-0)$ groups. Vertical lines (I) represent SD's and asterisks $(*)$ indicate a significant difference $(p<0.05)$ from the value before the glucose load

\section{Retention of $A G$ in circulation}

To study further the excretion of $\mathrm{AG}$ into the urine and its alteration in diabetes, the concentrations of $\mathrm{AG}$ and glucose in urine as well as the blood glucose and plasma AG were measured before and after a $100-\mathrm{g}$

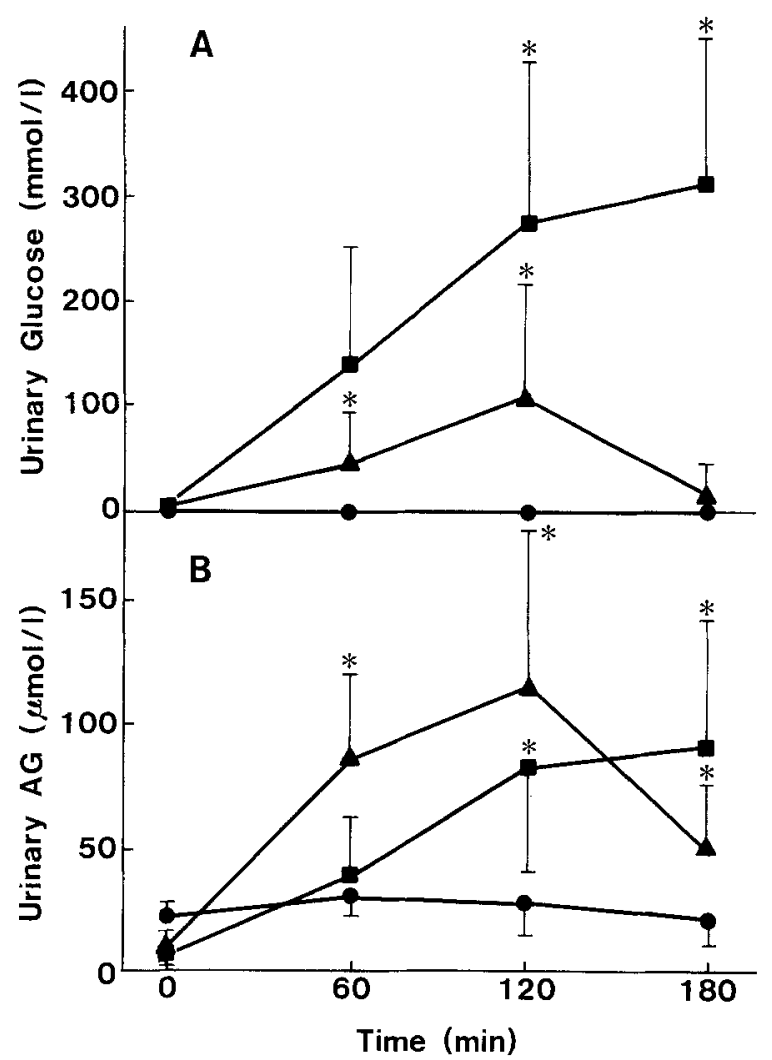

Fig.4. Effect of 100-g glucose load on the concentrations of glucose $(A)$ and $A G(B)$ in urine. Subjects and symbols are the same as those in Figure 3

oral glucose load in the control, IGT and diabetic groups. The average values of these measurements are shown in Figures 3 and 4. The plasma AG concentration before the glucose load was highest in the control group, intermediate in the IGT group and extremely low in the diabetic group. Similarly, the urinary AG concentration before the glucose load was highest in the control subjects, intermediate in the IGT subjects and lowest in the diabetic patients. However, these urinary concentrations were less than the corresponding plasma AG concentration in these three groups. Although Figure 4 presents the average values for the respective groups, the urinary AG concentration in each individual was roughly one-sixth of the corresponding plasma concentration in the control and IGT groups, and one-half as much as the plasma concentration in the diabetic group.

\section{Change in urinary concentrations of $A G$ and glucose after glucose load}

The results in Figures 3 and 4 also indicate an intimate correlation between urinary $A G$ and urinary glucose. The blood glucose responded to the glucose load in a manner typical of the respective categories: in the IGT and diabetic groups, the blood glucose generally increased more than 2-fold, showing an early recovery in the IGT group and no tendency of 
recovery within $180 \mathrm{~min}$ in the diabetic patients. On the other hand, in the control group, the blood glucose concentration after the glucose load was elevated slightly, but significantly $(p<0.05)$, and soon returned to the basal concentration. The urinary AG and glucose responded similarly to the change in the blood glucose concentration after the glucose load in the IGT and diabetic groups. In the IGT group, dramatic transient increases in both $A G$ and glucose in the urine, more than 10 -fold for $A G$ and to a very variable extent (from 10 -fold to 500 -fold) for glucose, accompanied the temporal change in the blood glucose concentration, which transiently exceeded the threshold for renal glucose excretion. In the diabetic group, the two elevated urinary concentrations were as lasting as the blood glucose concentration and never recovered within the observation time. In the control group, the two urinary concentrations after glucose load did not significantly change during the observation period. In contrast to the change in the AG concentration in the urine, the plasma AG concentration was stable and hardly responded to the glucose load even in the IGT and diabetic groups, in spite of the fact that a marked $A G$ excretion was observed in these groups.

\section{Discussion}

This study demonstrated the general presence of $A G$ in human urine. The AG amount in 24-h urine samples ranged from 0 to $60 \mu \mathrm{mol}$, which was much smaller than the AG amount in the circulation of the subjects in the control group, estimated to be more than $450 \mu \mathrm{mol}$ (assuming the total blood volume to be 51 ). This indicates that $A G$ is largely retained in the circulation and only partly excreted in the urine under normal conditions. In the diabetic group, the 24-h urinary AG showed an appreciable correlation with the urinary glucose. This observation suggests that AG excretion might be promoted by a high blood glucose concentration. This concept was further supported by a glucose tolerance test demonstration that elevation of the urinary $A G$ concentration was closely related to elevation of the blood glucose concentration in manner similar to the urinary glucose concentration.

The plasma AG concentration was relatively constant during the glucose tolerance test, even in the IGT and diabetic patients who showed appreciably high $A G$ concentrations in the urine during the test. This could be explained if there was still a large AG pool in these patients relative to the excreted AG amount. In our previous study [12], we postulated a large AG pool in the human body since we had demonstrated that the $A G$ pool in normal rats was 6-fold larger than the AG pool in the circulation. In a recent study, we have also demonstrated that the major portion of that large $A G$ pool in the normal rat is excreted into the urine upon induction of an acute and drastic increase in the blood glucose concentration either by treatment with streptozocin or alloxan, or by intravenous infusion of glucose (unpublished data). In these experiments, the AG excretion was accompanied by a marked decrease in the plasma AG concentration. The large AG pool we postulate in humans by analogy with rats would be significantly depleted only by a drastic and/or persistent high glucose concentration. The diurnal stability of the plasma AG concentration reported earlier [11] would also support the presence of a considerably large AG pool in humans.

Although the origin and factors affecting the production and/or ingestion rate of $A G$ in the human body have not been clarified, it may be reasonable to conclude from the above considerations that enhanced and frequent urinary $A G$ excretion in diabetes would lead primarily to reduction of the plasma AG concentration.

The fact that such a small and hydrophilic molecule as AG is retained in the circulation indicates that AG is filtered out by the glomeruli and, accordingly, largely reabsorbed by the renal tubules. It also indicates that AG is reabsorbed like glucose by an active transporter which acts against the concentration gradient, because the basal concentration of urinary AG was demonstrated to be much lower than that of plasma AG in the control subjects. Since AG has a structure similar to glucose, it is possible that AG shares a transporter with glucose in the renal tubules. The concept of a common transporter for AG and glucose readily accommodates the present study's finding of marked promotion of AG excretion by a high blood glucose concentration. When the blood glucose concentration exceeds the threshold for glucose excretion, the tubular sites for glucose reabsorption may be saturated and only a small number of transporters may be left available for $A G$ reabsorption; consequently, the AG reabsorption may be reduced or abolished in this situation and AG would be excreted. Support for the plausibility of a common active transporter for $A G$ and glucose has been obtained in vitro [15]; AG was effectively transported by the glucose transporter of hamster intestine in an energy-linked manner. The idea of active AG transport also accommodates the efficient ingestion of orally-administered AG demonstrated in rats [8]. To date, we do not know the effect of glomerular function on the urinary AG excretion. The present study did not demonstrate any significant correlation between 24-h urinary AG excretion and plasma creatinine concentration in the patients with diabetes mellitus.

In summary, the present study demonstrated that AG was largely retained in the circulation of nondiabetic control subjects and that an elevation of renal AG excretion accompanied renal glucose excretion. The plasma AG concentration appeared to be reduced by frequent and/or persistent excretion of AG. The 
present findings also suggest that AG may share tubular transporters with glucose. This concept provides a plausible mechanism which accounts for the diabetesspecific low plasma $A G$ concentration.

\section{References}

1. Pitkänen E (1973) Occurrence of 1,5-anhydroglucitol in human cerebrospinal fluid. Clin Chim Acta 48: 159-166

2. Servo C, Pitkänen E (1975) Variation in polyol levels in cerebrospinal fluid and serum in diabetic patients. Diabetologia 11: $575-580$

3. Servo C, Palo J, Pitkänen E (1977) Polyols in the cerebrospinal fluid and plasma of neurological, diabetic and uremic patients. Acta Neurol Scand 56: 111-116

4. Akanuma H, Ogawa K, Lee Y, Akanuma Y (1981) Reduced levels of 1,5-anhydroglucitol in diabetic patients. J Biochem 90: 157-162

5. Pitkänen E (1982) Serum 1,5-anhydroglucitol in normal subjects and in patients with insulin-dependent diabetes mellitus. Scand $\mathbf{J}$ Clin Lab Invest 42: 445-448

6. Yoshioka S, Fujisawa T, Fujimori A, Takatani O, Funabashi M (1982) Identification and metabolic implication of 1-deoxyglucose (1,5-anhydroglucitol) in human plasma. Clin Chem 28: 1283-1286

7. Yoshioka S, Saitoh S, Negishi C, Fujisawa T, Fujimori A, Takatani O, Imura M, Funabashi M (1983) Variation of 1-deoxyglucose (1,5-anhydroglucitol) content in plasma from patients with insulin-dependent diabetes mellitus. Clin Chem 29: 1396-1398

8. Pitkänen E, Pitkänen $O$ (1984) The elimination of 1,5-anhydroglucitol administered to rats. Experientia 40: 463-465
9. Yamanouchi T, Akanuma H, Takaku F, Akanuma Y (1986) Marked depletion of plasma 1,5-anhydroglucitol, a major polyol, in streptozocin-induced diabetes in rats and the effect of insulin treatment. Diabetes 35: 204-209

10. Yamanouchi T, Akanuma H, Nakamura T, Akaoka I, Akanuma Y (1988) Specific reduction of plasma 1,5-anhydroglucitol (1-deoxyglucose) concentration in diabetic patients. Diabetologia 31: 41-45

11. Yamanouchi T, Akanuma H, Asano T, Konishi C, Akaoka I, Akanuma Y (1987) Reduction and recovery of plasma 1,5-anhydro-D-glucitol in diabetes mellitus. Diabetes 36: 709-715

12. Kametani S, Hashimoto Y, Yamanouchi T, Akanuma Y, Akanuma H (1987) Reduced renal reabsorption of 1,5-anhydro-D-glucitol in diabetic rats and mice. J Biochem 102: 1599-1607

13. WHO study group (1985) In "Diabetes Mellitus" (Technical report series 727). World Health Organization, Geneva

14. Ohkubo A, Kamei S, Yamanaka M, Arai F, Kitajima M, Kondo A (1981) Plasma glucose concentrations of whole blood, as determined with a multilayer-film analytical element. Clin Chem 27: $1287-1290$

15. Crane RT, Mandelstam P (1960) The active transport of sugars by various preparations of hamster intestine. Biochim Biophys Acta $45: 430-475$

Received: 28 December 1987

and in revised form: 19 September 1988

Dr. H. Akanuma

Department of Chemistry

College of Arts and Sciences

University of Tokyo

Meguro-ku, Tokyo, 153

Japan 